\title{
Inhibition of the Glycine Cleavage System by Branched-Chain Amino Acid Metabolites
}

\author{
STEEN KØLVRAA \\ Research Laboratory for Metabolic Disorders, University Department of Clinical Chemistry, Aarhus \\ Kommunehospital, Denmark
}

\section{Summary}

The effects of 18 normally occurring and 11 patalogical metabolites of the branched-chain amino acids on the glycine cleavage system were investigated on intact rat liver mitochondria. It was demonstrated, that 2-oxo-isovaleric acid, 2-methyl-butyric acid, and isobutyric acid significantly inhibited the glycine cleavage system in intact mitochondria.

Further studies on the solubilized glycine cleavage system demonstrated that the inhibitory effect was due to 2 -methyl-butyrylCoA (linear noncompetitive inhibition, $\mathrm{k}_{\mathrm{i}}: 0.1-0.15 \mathrm{mM}$ ) and isobutyryl-CoA (S-hyperbolic, I-linear noncompetitive inhibition, $\mathbf{k}_{\mathrm{i}}$ : 0.2-0.3 mM). Both 2-methyl-butyric acid and isobutyric acid exhibited less inhibition (2-methyl-butyric acid: competitive inhibition, $k_{i}: 5.5 \mathrm{mM}$, isobutyric acid: competitive inhibition, $k_{i}: 16$ $\mathrm{mM}$ ), while 2-oxo-isovaleric acid was without inhibitory effect, and probably affects intact mitochondria through transformation to isobutyryl-CoA.

It is suggested that the inhibitory action of 2-methyl-butyryl$\mathrm{CoA}$ and isobutyryl-CoA may explain the hyperglycinemia seen in propionyl-CoA carboxylase deficiency, methyl-malonyl-CoA mutase deficiency and $\beta$-ketothiolase deficiency.

\section{Speculation}

In patients not suffering from any known inborn error of metabolism, hyperglycinemia has been described in connection with two circumstances, namely, severe generalized illness and medication with dipropylacetic acid. Because both these conditions might cause some derangement of the branched-chain amino acid metabolism, it is speculated that this hyperglycinemia might be due to inhibition of the glycine cleavage system by 2-methyl-butyryl-CoA and isobutyryl-CoA, and that these conditions thus might serve as models for ketotic hyperglycinemia.

In 1961, Childs and Nyhan (4) described a syndrome, named the hyperglycinemia syndrome, presenting as a severe neonatal illness in a child with elevated glycine concentrations in both serum and urine. In a following paper (23), they showed that administration of branched-chain amino acids resulted in deterioration of the clinical symptoms and further elevation of the glycine concentrations.

During the following years, several new cases of hyperglycinemia were reported, and it became obvious that symptoms and signs associated with this condition varied considerably. It was, therefore, proposed that the syndrome covered several ethiologic entities (9).

It is now firmly documented that the condition exists in connection with at least five different enzymatic defects. Three are in the metabolism of branched-chain amino acids (propionyl-CoA carboxylase deficiency (2), methylmalonyl-CoA mutase deficiency (18), and $\beta$-ketothiolase deficiency (11), and one in the metabolism of glycine itself, namely, deficiency of the glycine cleavage system
(24). The fifth has recently been described in a child with deficient D-glycerate dehydrogenase activity (16).

Whereas it is not surprising that a primary defect in the glycine cleavage system results in hyperglycinemia, it is still not clear why elevated glycine concentrations in serum and urine are a feature of disorders in the metabolism of the branched-chain amino acids, and of D-glyceric acid.

In cases of propionyl-CoA carboxylase deficiency and methylmalonyl-CoA mutase deficiency, where hyperglycinemia was a feature, diminished glycine cleavage activity in liver tissue has been demonstrated. This finding is in addition to the primary defects $(6,22)$, but its significance remains unclear at present.

Investigations of a possible intercorrelation between metabolites of the branched-chain amino acids, and glycine metabolism were, therefore, undertaken. Results presented here indicate that 2methyl-butyric acid and isobutyric acid, and especially their CoA derivatives, have inhibitory effects on the glycine cleavage system.

\section{MATERIALS AND METHODS}

\section{CHEMICALS}

L-isoleucine, 3-hydroxy-3-methyl-glutaric acid, 2-oxo-isocapronic acid, 2-oxo-isovaleric acid, 2-oxo-3-methyl-valeric acid, and pyridoxal phosphate were obtained from Sigma Chemical Company, St. Louis, MO., USA. Propionic acid, isobutyric acid, and isovaleric acid were obtained from BDH Chemicals Ltd., England. Methacrylic acid, D,L-2-methyl-butyric acid, tiglic acid, citraconic acid, glycine, and folic acid were obtained from $E$. Merck, Darmstadt, W. Germany. Acrylic acid and 3-amino-isobutyric acid were obtained from Koch-Light Laboratories Ltd., England. 3,3-dimethyl-acrylic acid was obtained from Aldrige Chemical Co., Inc., USA. Methylmalonic acid was obtained from Fluka AG, Switzerland. 3-methyl-glutaconic acid methyl ester and 3-hydroxy-propionic acid were obtained from EGA Chemie, W. Germany. 1-leucine and L-valine were obtained from La Rosch and Co., Switzerland. $\beta$-adenine dinucleotide $\left(\mathrm{NAD}^{+}\right)$ was purchased from Boehinger Mannheim GmbH, W. Germany. ${ }^{14} \mathrm{C}$-l-glycine was obtained from NEN Chemicals, W. Germany. 3-methyl-glutaconic acid was synthesized by mild alkaline hydrolysis of the corresponding ester. 2-methyl-3-hydroxy-butyric acid was synthesized as previously described (15). 3-hydroxyisobutyric acid was synthesized from 3-amino-isobutyric acid after the method of Landaas (17). 3-hydroxy-isovaleric acid was synthesized from 3,3-dimethyl-acrylic acid after the method of Pressman and Lucas (25). After synthesis, all the acids were purified by chromatography on silicic acid columns as described by Kesner and Muntwyler (14).

The CoA-derivatives of D,L-2-methyl-butyric acid and isobutyric acid were synthesized from the respective acid-chlorides (28). Reaction products were desalted on a column of G-10 Sephadex and the CoA-derivatives then purified on a A-25 Sephadex column. Glycine conjugates of isovaleric acid, D,L-2-methyl-butyric 
acid, isobutyric acid, 3,3-dimethyl-acrylic acid, tiglic acid, methacrylic acid, and propionic acid were synthesized according to Bondi and Eissler (3). Tetrahydrofolic acid was synthesized from folic acid after the method of Davis (7).

\section{ISOLATION OF MITOCHONDRIA}

2-3 month-old rats of Wistar strain (150-200 g) were stunned with a blow to the head and killed by spinal dislocation. The liver was immediately removed, chilled on ice, and mitochondria isolated according to Schneider and Hogeboom (26) in a buffer containing $225 \mathrm{mM}$ mannitol, $25 \mathrm{mM}$ sucrose, $1 \mathrm{mM}$ Tris- $\mathrm{HCl}$, $0.1 \mathrm{mM}$ EDTA ( $\mathrm{pH}: 8.1$ ). The mitochondria used had respiratory control ratios of over 5 , judged on a Clark-type oxygen electrode using pyruvate as substrate.

\section{PREPARATION OF SOLUBILIZED GLYCINE CLEAVAGE SYSTEM}

The mitochondrial suspension was mixed with 10 volumes of acetone precooled to $-20^{\circ}$, and left at $-20^{\circ}$ for $10 \mathrm{~min}$. The precipitate was washed several times with cooled acetone and dried in vacuo. The residue was dissolved in 20 volumes of 0.002 Tris- $\mathrm{HCl}(\mathrm{pH}: 8.1)$ and left with continuous stirring for $30 \mathrm{~min}$ at $4^{\circ}$. This solution was then centrifuged at $15.000 \mathrm{~g}$ for $10 \mathrm{~min}$, and the supernatant was dialyzed against a large volume of the same buffer followed by lyophilization. The resulting powder could be stored at $-20^{\circ}$ for several months without loss of activity.

\section{GLYCINE CLEAVAGE ACTIVITY IN INTACT MITOCHONDRIA}

Freshly prepared mitochondria and cofactors were incubated at $37^{\circ}$ with ${ }^{14} \mathrm{C}$-l-glycine for $30 \mathrm{~min}$. The reaction was started by simultaneous addition of tetrahydrofolic acid and glycine, and terminated by addition of $0.2 \mathrm{ml} 5 \mathrm{~N}$ sulphuric acid. Any $\mathrm{CO}_{2}$ produced during the incubation was trapped by the method of Gliemann (10). Scintilation fluid was added and ${ }^{14} \mathrm{C}$-activity counted in a liquid-scintilation counter. Reaction mixture: 20 $\mu$ mole ${ }^{14} \mathrm{C}-1$-glycine (specific activity: $0.5 \mathrm{mCi} / \mathrm{mmole}$ ), $2.5 \mu \mathrm{mole}$ dithiotreitol, $1.9 \mu$ mole pyridoxal phosphate, $8.6 \mu$ mole $\mathrm{NAD}^{+}$, $0.75 \mu$ mole tetrahydrofolic acid, inhibitors in concentrations as listed in Table 1, mitochondria equivalent to $2.5 \mathrm{mg}$ protein, 67.5 $\mu$ mole Tris- $\mathrm{HCl}$ (pH:8.1), total volume $1.35 \mathrm{ml}$.

\section{GLYCINE CLEAVAGE ACTIVITY IN THE SOLUBILIZED SYSTEM}

Incubations were done essentially as described above. Reaction mixture: $5-20 \mu$ mole ${ }^{14} \mathrm{C}$-l-glycine (specific activity: $1.5 \mathrm{mCi}$ / mmole), $4 \mu$ mole dithiotreitol, $0.95 \mu$ mole pyridoxal phosphate, $1.75 \mu$ mole $\mathrm{NAD}^{+}, 0.75 \mu$ mole tetrahydrofolic acid, inhibitors in varying concentrations, $1-2 \mathrm{mg}$ protein, $67.5 \mu$ mole Tris- $\mathrm{HCl}(\mathrm{pH}$ : 8.1 ), total volume $1.35 \mathrm{ml}$. $\mathrm{CO}_{2}$ was trapped and counted as described above.

\section{RESULTS}

\section{PROPERTIES OF THE GLYCINE CLEAVAGE SYSTEM}

In preliminary experiments, the glycine cleavage activity in the two systems were optimized with respect to concentrations of cofactors and $\mathrm{pH}$. The reaction proceeded linearly for at least 30 min and the velocity increased linearly up to $5 \mathrm{mg}$ protein (mitochondria) and $7 \mathrm{mg}$ protein (soluble system). The $\mathrm{k}_{\mathrm{m}}$, with respect to glycine, was $8-10 \mathrm{mM}$ in both systems, which means that the substrate concentrations used could not be fully saturated. $\mathrm{V}_{\max }$ was $8.6 \mathrm{nmole} / \mathrm{mg}$ protein $/ \mathrm{min}$ in the system using fresh mitochondria, and $0.9 \mathrm{nmole} / \mathrm{mg}$ protein $/ \mathrm{min}$ when using the solubilized system.

\section{INHIBITION OF INTACT MITOCHONDRIA}

Figure 1 shows the metabolic pathways for degradation of the branched-chain amino acids, together with the known and assumed side reactions that might occur in the metabolic disorders in question. These include formation of 3-hydroxy-isovaleric acid from isovaleric acid, formation of citraconic acid from tiglic acid and formation of acrylic acid and 3-hydroxy-propionic acid from propionic acid. All compounds in Figure 1 were tested (as the free acids) except 2-methyl-3-oxo-butyric acid and methylmalonyl semialdehyde. In addition, the glycine conjugates of isovaleric acid, D,L-2-methyl-butyric acid, isobutyric acid, 3,3-dimethylacrylic acid, tiglic acid, methacrylic acid, and propionic acid were tested. Investigations were performed with inhibitors at concentrations of $0.3,1.0,3.0$, and $10.0 \mathrm{mM}$ on two different rats for each compound.

The results of these studies showed that only three compounds,

Table 1. The effect of 2-oxo-isovaleric acid, 2-methyl-butyric acid, and isobutyric acid on the glycine cleavage system in fresh rat liver mitochondria. ${ }^{1}$

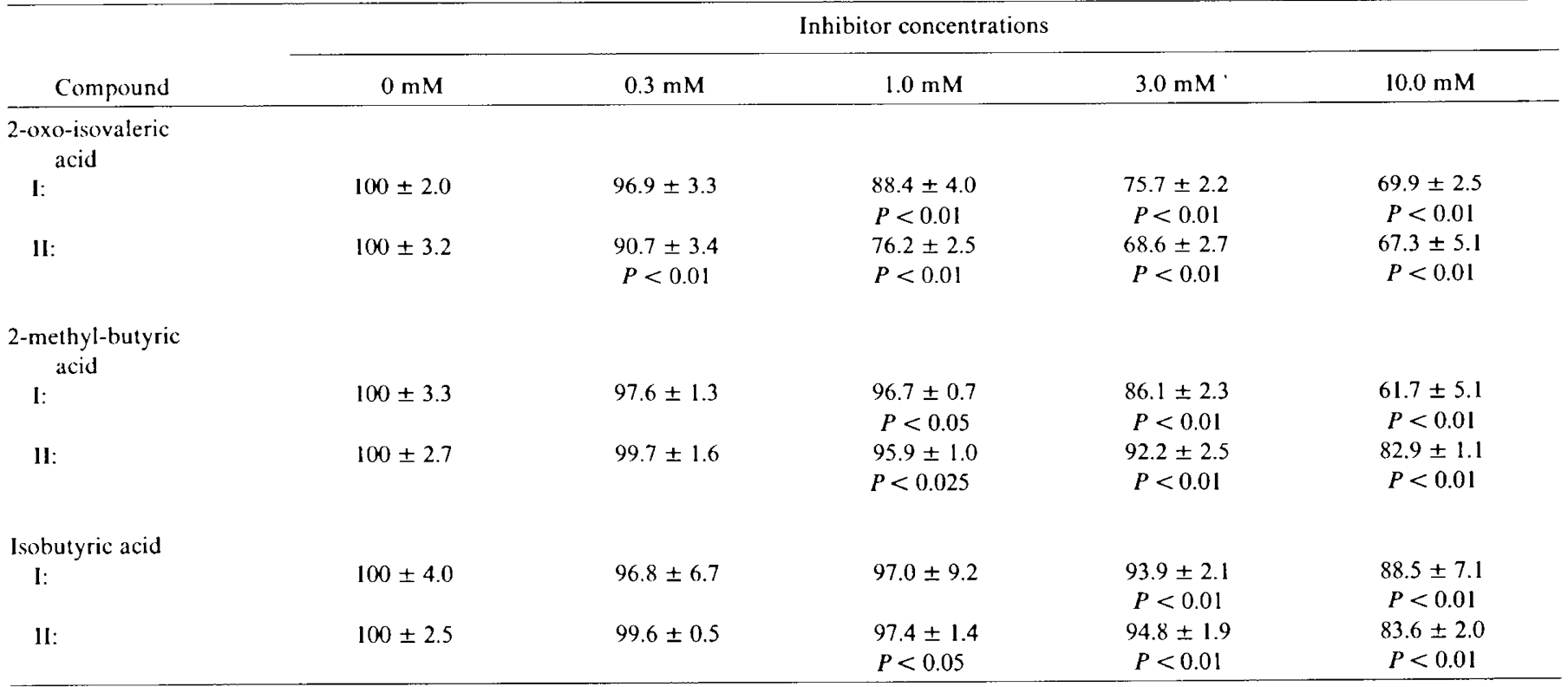

\footnotetext{
' Results are expressed as percent of the mean ${ }^{14} \mathrm{CO}_{2}$ production in assays without addition. Each result is mean \pm SD for five measurements. Each compound was tested on two different rats (marked I and II). The Student's $t$ test was used to compare results with various concentrations of inhibitor with results with no addition. Where no $P$ value is given, it was greater than 0.05 , i.e., no significant inhibition. The oxidation rate of ${ }^{14} \mathrm{C}$-1-glycine ranged in the six experiments without inhibitor from $3.4-4.5 \mathrm{nmole} / \mathrm{mg}$ protein $/ \mathrm{min}$.
} 


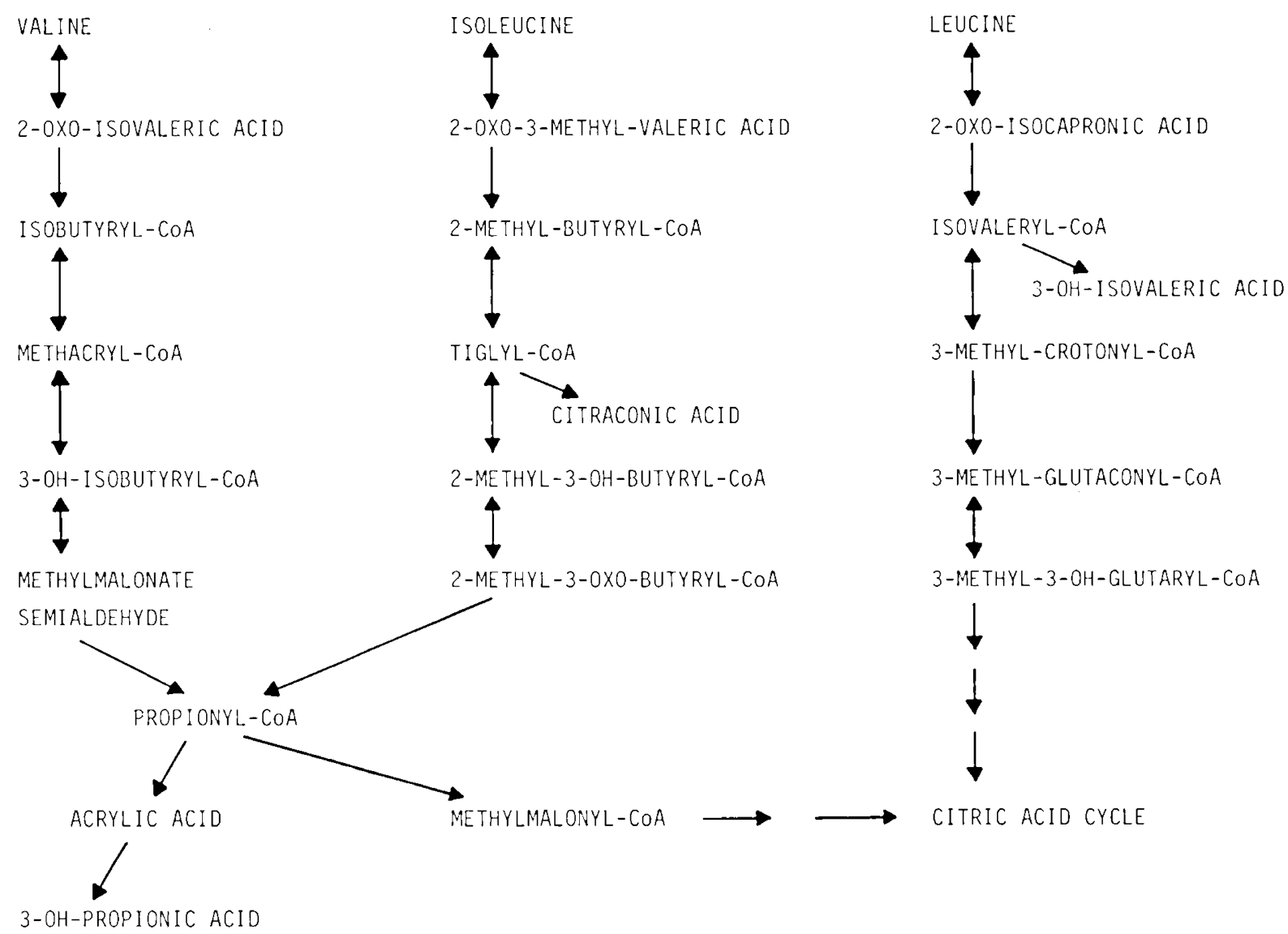

Fig. 1. The degradation pathways for valine, isoleucine, and leucine. All compounds shown except 2-methyl-3-oxo-butyric acid and methylmalonyl semialdehyde were tested for inhibitory action on fresh mitochondria (eventually as free acids).

namely, 2-oxo-isovaleric acid, 2-methyl-butyric acid, and isobutyric acid showed a consistent decrease in enzyme activity with increasing inhibitor concentration (Table 1). All other compounds showed either constant activity with increasing inhibitor concentration or a slight increase (data not shown). Furthermore, it is evident that while intraindividual determinations gave high levels of reproducibility, there was some interindividual variation in sensitivity towards the inhibitors.

\section{INHIBITION OF THE SOLUBILIZED GLYCINE CLEAVAGE SYSTEM}

The three metabolites shown to inhibit intact mitochondria were selected for further study in order to characterize their effect. This was done in solubilized enzyme preparation to obtain kinetic constants and to eliminate errors due to membrane transport. Because 2-methyl-butyric acid and isobutyric acid are normally predominantly CoA-derivatized within the mitochondria, both the free acids and the thiolesters were tested. Corresponding values of substrate concentrations, inhibitor concentrations, and initial velocities were obtained and analyzed in double reciprocal plots. The type of inhibition and the inhibitor constants found for the five compounds are shown in Table 2. In addition, the reciprocal plots are shown for 2-methyl-butyryl-CoA (Figure 2) and isobutyryl-CoA (Figure 3).

\section{DISCUSSION}

The properties of the glycine cleavage system measured in this study are in close agreement with those described by Motokawa and Kikuchi (20). The rather high $K_{m}(8-10 \mathrm{mM})$ is unusual but quite possible, because the intracellular glycine concentration in liver tissue is about $7 \mathrm{mM}(5)$. The very pronounced fall in $V_{\max }$ during the solubilisation procedure is not surprising, because Motokawa and Kikuchi (19) have shown that the glycine cleavage system is located on the inner mitochondrial membrane, probably as a complex. Acetone treatment probably disrupts this proteincomplex resulting in the marked reduction in $\mathrm{V}_{\text {max }}$ without altering $\mathrm{K}_{\mathrm{m}}$.

In the present communication, it has been shown that two metabolites of the branched-chain amino acids, namely the CoAderivatives of 2-methyl-butyric acid and isobutyric acid, inhibited the glycine cleavage system strongly. The corresponding free acids exhibited a much weaker inhibitory effect, while the apparent effect of 2-oxo-isovaleric acid was probably due to its transformation to isobutyryl-CoA during incubation.

Figure 1 shows that 2-methyl-butyryl-CoA and isobutyryl-CoA are located in the degradation pathways of isoleucine and valine, respectively, and exhibit analogue structural characteristics both being 2-methyl-substituted, short-chain fatty acids. No inhibition was demonstrated when testing metabolites from the degradation pathway of leucine. This is in agreement with the fact that no 2methyl-substituted compounds occur in this pathway, and that hyperglycinemia in connection with defects in the degradation of branched-chain amino acids has so far only been demonstrated with certainty in connection with defects in the degradation of valine and isoleucine (propionyl-CoA carboxylase deficiency, methylmalonyl-CoA mutase deficiency, and $\beta$-ketothiolase deficiency). A single publication describing slightly elevated glycine concentration in connection with isovaleric acidemia exists (1), but this has not since been confirmed in other patients with this disorder, and the hyperglycinemia described was atypical on several points. Whether the inhibitory effect of 2-methyl-butyrylCoA and isobutyryl-CoA can play a patogenetic role in ketotic hyperglycinemia (propionyl-CoA carboxylase deficiency, methylmalonyl-CoA mutase deficiency, and $\beta$-ketothiolase deficiency) is at present uncertain, because no information about intramitochondrial concentrations of these compounds in metabolic disorders is available. However, the presence of free isobutyric acid in serum and hydrolyzed urine from patients with propionyl-CoA 
Table 2. Inhibition pattern of the solubilized glycine cleavage system

\begin{tabular}{|c|c|c|c|}
\hline Compound & Type of inhibition & $\mathbf{K}_{\text {is }}$ & $\mathbf{K}_{\mathrm{ii}}$ \\
\hline 2-oxo-isovalerate & Mixed & $>40 \mathrm{mM}$ & \\
\hline 2-Me-butyrate & Competitive & $5.5 \mathrm{mM}$ & \\
\hline Isobutyrate & Competitive & $16 \mathrm{mM}$ & \\
\hline Isobutyryl-CoA & $\begin{array}{l}\text { S-hyperbolic-I-linear non- } \\
\text { competitive }\end{array}$ & & $0.2-0.3 \mathrm{mM}$ \\
\hline
\end{tabular}

\footnotetext{
I I, L-form.
}

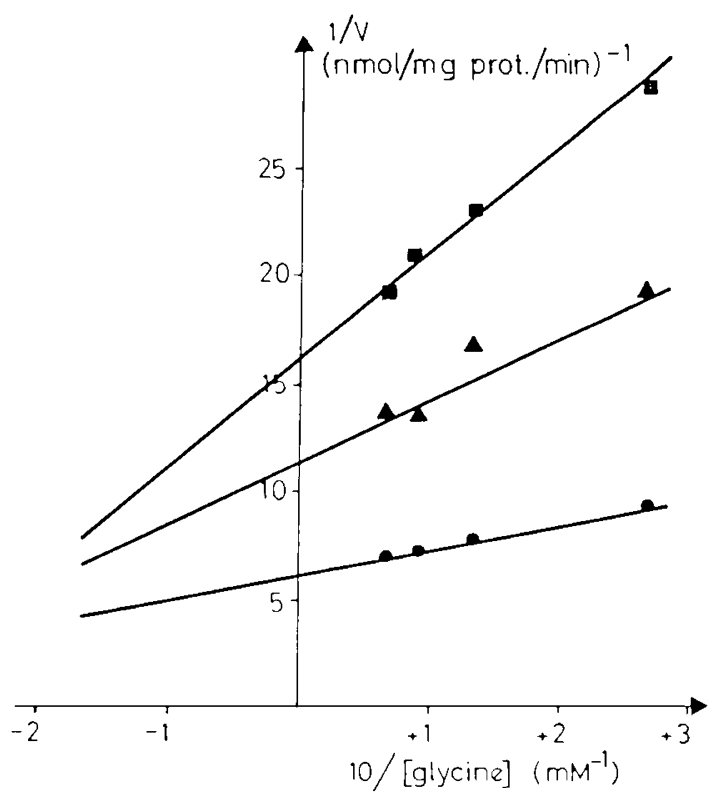

Fig. 2. Inhibition of the glycine cleavage system by 2-methyl-butyryl$\mathrm{CoA}$. The inhibition was measured with respect to glycine (glycine concentrations: $15,11.25,7.5,3.75 \mathrm{mM}$ ). The conditions for the assay of the solubilized system are described in Methods. $1 \mathrm{mM}$ 2-methylbutyryl-CoA, $-\mathbf{A} 2 \mathrm{mM}$ 2-methyl-butyryl-CoA, $-2 \mathrm{mM} 2$ methyl-butyryl-CoA.

carboxylase deficiency $(2,8)$, might support the hypothesis that an intramitochondrial accumulation of the thiolesters exists.

Until now only two other groups of workers have investigated the mechanism behind the diminished glycine cleavage activity seen in ketotic hyperglycinemia.

Hillman et al. (13) have studied the production of ${ }^{14} \mathrm{CO}_{2}$, when incubating cultured skin fibroblasts from a patient with $\beta$-ketothiolase deficiency and from normal controls with $\mathrm{U}-{ }^{14} \mathrm{C}$-glycine. The effect of preincubation with isoleucine was studied in detail, and it was shown that isoleucine incubation caused significant inhibition of ${ }^{14} \mathrm{CO}$-production in cells from the patient. These findings are of course quite compatible with the hypothesis proposed in the present communication. However, we have not been able to repeat these experiments, because of inability to demonstrate significant ${ }^{14} \mathrm{CO}_{2}$-production, when incubating ${ }^{14} \mathrm{C}$-l-glycine and ${ }^{14} \mathrm{C}-2$-glycine with cultured fibroblasts. Hillman et al. (13) suggested that earlier similar failures were due to the use of Eagles minimal essential medium. However, even when using F 10 , the same medium as that of Hillman et al. we were unable to detect significant activity. In a later publication (12), the same group studied the glycine/serine interconversion in a similar set-up and demonstrated that incubation with both isoleucine and tiglic acid inhibited this reaction, and they suggested that the inhibition of serine hydroxymethyl-transferase caused ketotic hyperglycinemia. However, this is in disagreement with several earlier publications $(6,27,29)$, which all demonstrated that there are deficiencies of the glycine cleavage system and not of serine hydroxymethyltransferase in patients with ketotic hyperglycinemia. Therefore, it must be concluded that it is impossible to determine to what

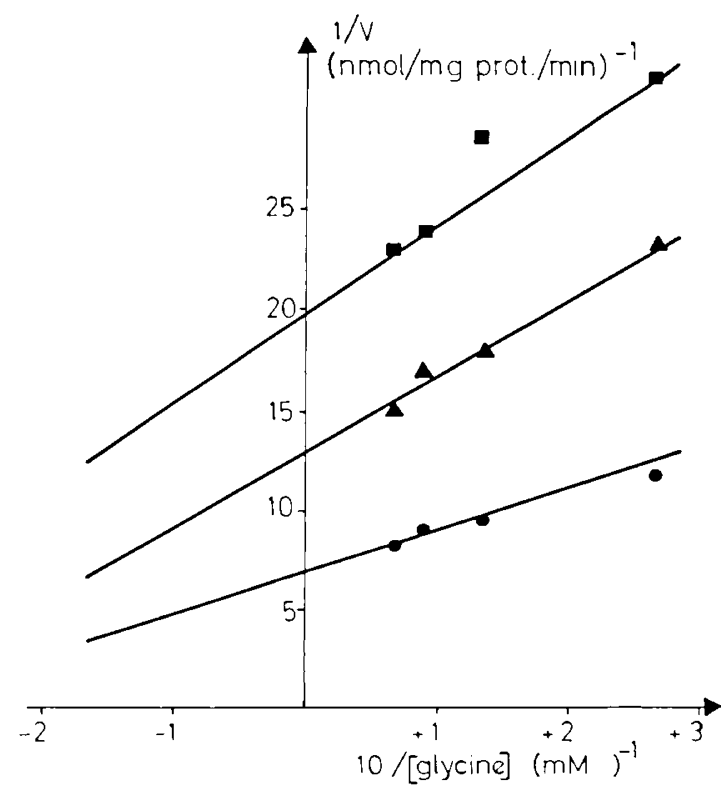

Fig. 3. Inhibition of the glycine cleavage system by isobutyryl-CoA. The inhibition was measured with respect to glycine (glycine concentrations: $15,11.25,7.5$, and $3.75 \mathrm{mM}$ ). The conditions for the assay of the solubilized system are described in Methods. $1 \mathrm{mM}$ isobutyrylCoA, $\Delta-\mathbf{\Delta} 2 \mathrm{mM}$ isobutyryl-CoA, $-3 \mathrm{mM}$ isobutyryl-CoA

extent the experiments of Hillman et al. (12) offer support to the hypothesis put forward in the present communication.

Recently, Motokawa et al. (21) published a work on the concentrations of the individual protein components of the glycine cleavage system in liver tissue from patients with ketotic hyperglycinemia. These concentrations were measured indirectly, by adding to the reaction mixture excess quantities of all the other components, obtained from animal and bacterial sources, and then using the resulting enzyme activity as a measurement of the concentration. Using this method, they demonstrated that the concentration of $\mathrm{P}-, \mathrm{T}$ - and $\mathrm{H}$-protein in liver tissue from patients amounted to only $30-50 \%$ of normal. In addition, they showed that when purifying the $\mathrm{H}$-protein from both patient and control, essentially the same specific activity of $\mathrm{H}$-protein was obtained, and that the activity/g wet weight was 3.5 times lower in the patient than in the control. Based on these findings, they concluded that the hyperglycinemia seen in patients with ketotic hyperglycinemia was due to diminished levels of glycine cleavage system in liver cells.

These findings point indirectly to inhibition of the glycine cleavage synthesis, rather than to inhibition of its activity. It is, however, difficult to interpret these results, especially whether enzyme components can be quantified as described, and it is difficult to judge in which way an enzyme inhibitor of a certain affinity will modify the results.

It is therefore concluded, that the hyperglycinemia seen in propionyl-CoA carboxylase deficiency, methylmalonyl-CoA mutase deficiency, and $\beta$-ketothiolase deficiency can be due to both inhibition of the glycine cleavage activity, and to inhibition of the synthesis of the glycine cleavage enzyme complex, and that no 
direct evidence favoring any of the explanations is available at present. The question can only be answered by direct measurement of either the synthesis of the glycine cleavage complex or of the intramitochondrial concentrations of thiolesters in patients with ketotic hyperglycinemia.

\section{REFERENCES AND NOTES}

1. Ando, T., Klingberg, W. G., Ward, A. N., Rasmussen, K., and Nyhan, W. L.: Isovaleric acidemia presenting with altered metabolism of glycine. Pediatr. Res., 5: 478 (1971).

2. Ando, T., Rasmussen, K.. Nyhan, W. L., Donnell, G. N., and Barnes, N. D.: Propionic acidemia in patients with ketotic hyperglycinemia. J Pediatrics, 78: 827 (1971).

3. Bondi, S., and Eissler, F.: Über lipoproteide und die Deutung der degenerativen Zellverfettung. Biochem. Zeith, 23: 499 (1916)

4. Childs, B., Nyhan, W. L., Borden. M., Bard, L., and Cooke. R. E.: Idiopathic hyperglycinemia and hyperglycinuria: A new disorder of amino acid metabolism I. Pediatrics, 27: 522 (1961).

5. Christensen, H. N.: Free amino acids and peptides in tissue. In: H. N. Munro and J. B. Allison: Mammalian Protein Metabolism. p. 105 (Academic Press, New York, 1964)

6. Corbeel, L. Tado, K., Colombo, J. P., Eeckels, R., Eggermont, E., Jaeken, J., Den Tandt, W., Harvengt, L., Delhaye, J., and Deloecker, W.: Methylmalonic acidemia and nonketotic hyperglycinemia. Arch. Dis. Childhood, 50: 103 (1975).

7. Davis, L.: A simple method for the synthesis of tetrahydrofolic acid. Analyt. Biochem., 26: 459 (1968).

8. Duran, M.: A contribution to the study of organic aciduria. Thesis, Utrecht (1974).

9. Gerritsen, T., Kaveggia, E., and Waisman H. A.: A new type of idiopathic hyperglycinemia with hypo-oxaluria. Pediatrics, 36: 882 (1965).

10. Gliemann, J.: Insulin-like activity of diluted human serum assayed by an isolated adipose cell method. Diabetes, 14: 643 (1965).

11. Hillman, R. E., and Keating, J. P.: $\beta$-ketothiolase deficiency as a cause of the ketotic hyperglycinemia syndrome. Pediatrics, 53: 221 (1974).

12. Hillman, R. E., and Otto. E. F.: Inhibition of glycine-serine interconvertion in cultured human fibroblasts by products of isoleucine catabolism. Pediatr. Res. 8: 941 (1974).

13. Hillman, R. E., Sowers, L. H., and Cohen, J. L.: Inhibition of glycine oxydation in cultured fibroblasts by isoleucine. Pediatr. Res., 7: 945 (1973).

14. Kesner, L.. and Muntwyler, E.: Automatic determination of weak organic acids by partition column chromatography and indicator titration. Anal. Chem. 38 : 1164 (1966).

Copyright (c) 1979 International Pediatric Research Foundation, Inc. $0031-3998 / 79 / 1308-0889 \$ 02.00 / 0$
15. Kolvraa, S., Gregersen. N., Christensen, E., and Gron, I.: Calcium levulinate medication. A pitfall in the diagnosis of organic acidurias. Clin. Chim. Acta, 77: 197 (1977).

16. Kalvraa, S. Rasmussen, K.. and Brandt. N. J.: I)-glyceric acidemia: biochemical studies in a new syndrome. Pediatr. Res., 10: 825 (1976).

17. Landaas, S.: Accumulation of 3-hydroxyisobutyric acid, 2-methyl-3-hydroxybutyric acid, and 3-hydroxyisovaleric acid in ketoacidosis. Clin. Chim. Acta, 64: 143 (1975).

18. Morrow, G., Barness, L. A.. Auerbach, V. H., DiGeorge, A. M., Ando, T., and Nyhan, W. L.: Observations on the coexistence of methylmalonic acidemia and glycinemia. J. Pediatrics, 74: 680 (1969).

19. Motokawa, Y., and Kikuchi, G.: Glycine metabolism in rat liver mitochondria, V. Arch. Biochem. Biophys., 146: 461 (1971).

20. Motokawa, Y.. and Kikuchi, G.: Glycine metabolism by rat liver mitochondria. Arch. Biochem. Biophys., I64: 624 (1974).

21. Motokawa, Y., Kikuchi, G., Narisawa, K., and Arakawa, T.: Reduced level of glycine cleavage system in the liver of hyperglycinemia patients. Clin. Chim. Acta, 79: 173 (1977).

22. Nishimira, $Y$. Tada, $K$. and Arakawa, $T$.: Coexistence of defective activity in glycine cleavage reaction and propionyl-CoA carboxylase in the liver of a hyperglycinemic child. Tohoku J. Exptl. Med., 113: 267 (1974).

23. Nyhan, W. L., Borden. B. A., and Childs, B.: Idiopathic hyperglycinemia: a new disorder of amino acid metabolism. Pediatrics, 27: 539 (1961).

24. Perry, T. L., Urquhart, N.. MacLean, J., Evans, M. E., Hansen, S., Davidson, G F., Applegarth, D. A., MacLeod, P. J., and Lock. J. E.: Nonketotic hyperglycinemia. N. Engl. J. Med., 292: 1269 (1975).

25. Pressman, D. and Lucas, H. J.: The hydration of unsaturated compounds. J. Am Chem. Soc., 62: 2069 (1940).

26. Schneider, W. C., and Hogeboom, G. H.: Intracellular distribution of enzymes V. J. Biol Chem., 183: 123 (1950).

27. Shafai, T., Sweetman, L., Weyler, W., Goodman, S. I., Fennessey, P. V., and Nyhan, W. L.: Propionic acidemia with severe hyperammonia and defective glycine metabolism. J. Pediatrics, 92: 84 (1978).

28. Stadiman, E. R. Preparation and assay of acyl coenzyme $A$ and other thiol esters, use of hydroxylamine. In: S. P. Colowick and N. O. Kaplan: Methods of Enzymology. Vol. 3, p. 931 (Academic Press, New York, 1957)

29. Tada, K., Corbeel, L. M., Eeckels, R., and Eggermont. E.: A block in glycine cleavage reaction as a common mechanism in ketotic and nonketotic hyperglycinemia. Pediatr. Res., 8: 721 (1974).

30. The authors acknowledge the assistance of Mrs. Inga Knudsen

31. This research was supported by a grant from the Danish Medical Research Council.

32. Requests for reprints should be addressed to: Steen Kølvraa, Department of Clinical Chemistry, Aarhus kommunehospital, DK-8000 Aarhus C, Denmark. 33. Received for publication May 22, 1978

34. Accepted for publication August 8, 1978. 